\title{
On Nonlinear Stability of General Undercompressive Viscous Shock Waves
}

\author{
Tai-Ping Liu, Kevin Zumbrun \\ Department of Mathematics, Stanford University, Stanford, CA 94305, USA
}

Received: 20 September 1994

\begin{abstract}
We study the nonlinear stability of general undercompressive viscous shock waves. Previously, the authors showed stability in a special case when the shock phase shift can be determined a priori from the total mass of the perturbation, using new pointwise methods. By examining time invariants associated with the linearized equations, we can now overcome a new difficulty in the general case, namely, nonlinear movement of the shock. We introduce a coordinate transformation suitable to treat this new aspect, and demonstrate our method by analyzing a model system of generic type. We obtain sharp pointwise bounds and $L^{p}$ behavior of the solution for all $p, 1 \leqq p \leqq \infty$.
\end{abstract}

\section{Contents:}

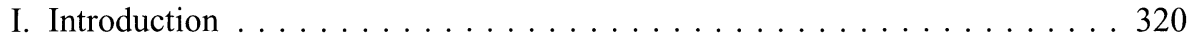

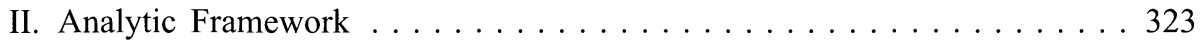

2.1. Preliminaries . . . . . . . . . . . . . . . . . . . . . . 323

2.2. Time Invariants of the Linearized Equation . . . . . . . . . . . 324

2.3. Linear Decay Criterion . . . . . . . . . . . . . . . . . . 326

2.4. Diagonalizing Transformation . . . . . . . . . . . . . . 328

2.5. Nonlinear Version . . . . . . . . . . . . . . . . . . . . . . . . . 329

III. Application to an Example System . . . . . . . . . . . . . 330

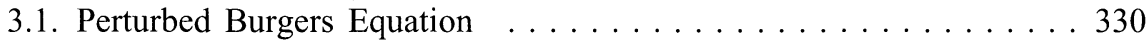

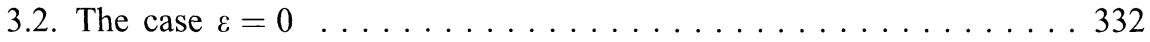

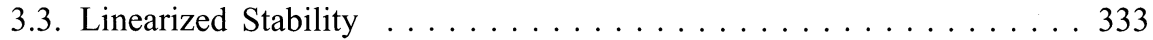

3.4. Nonlinear Stability . . . . . . . . . . . . . . . . . . . . . 335

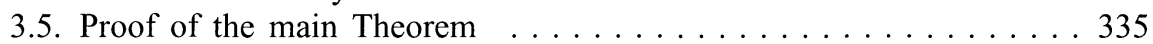

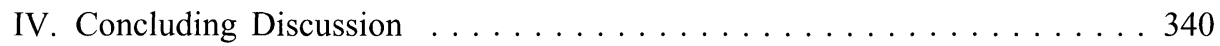

4.1. Generality . . . . . . . . . . . . . . . . . . . . . . . . . . 340

4.2. Curved Shocks . . . . . . . . . . . . . . . . . 340

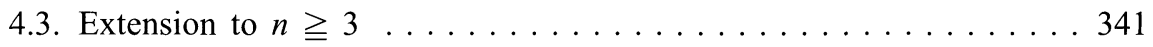

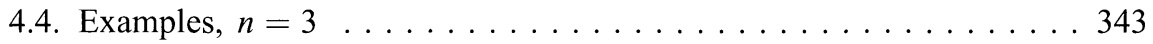




\section{Introduction}

An undercompressive shock of an $n \times n$ system of conservation laws is a viscous profile which has less than the usual $n+1$ entering (i.e. compressive) characteristics, violating Lax' condition for linearized stability. Such waves arise as solutions of conservation laws near regions where the classic assumption of strict hyperbolicity breaks down for example in petroleum reservoir models, or other models of multiphase flow [Ma-Me, Sch-Sh].

Undercompressive shocks have been shown to be generic features in solutions of Riemann problems of $2 \times 2$ systems with quadratic flux functions [I-Ma-Pl, Sch-Sh], which have been proposed as canonical models for behavior of general systems near a point of nonstrict hyperbolicity. Despite violating the classical Lax characteristic condition for linearized hyperbolic stability, these shocks appear to be stable when dissipative effects are included, [Zu-Pl-Ma, $\mathrm{L}-\mathrm{Zu}]$, even though their existence and profile depend sensitively on the form of the viscosity matrix. (Overcompressive shocks have distinct behavior, [L4, F-L].)

This illustrates an important point. The stability, and thus the physical admissibility of nonclassical shock waves cannot be ascertained by hyperbolic considerations, but rather must be studied in the context of a parabolic system including viscosity. The question of physical admissibility is particularly important in the case of undercompressive shocks, since they are a necessary component of solutions to Riemann problems; their inadmissibility would mean failure of hyperbolic theory to approximate small-viscosity behavior. Their admissibility, on the other hand, means that it is possible to have a hyperbolic theory which depends on the viscosity matrix of the encompassing viscous system. These considerations make imperative the careful analysis of nonlinear stability of viscous undercompressive shock waves.

Here, we study the behavior under perturbation of an undercompressive travelling wave solution, $\Phi(x-s t)$ of a parabolic system of conservation laws

$$
U_{t}+f(U)_{x}=\left(D(U) U_{x}\right)_{x}, \quad U \in R^{n},
$$

where the matrix $D(U)$ is positive definite. Though there is numerical evidence for the stability of a wide range of such shocks [Zu-Ma-Pl], a rigorous analysis of nonlinear stability has only been carried out in a single, nongeneric case [L-Zu]. In the present paper, we develop an analytic framework for the study of general undercompressive waves, then illustrate the method by the complete analysis of a particular system of generic type. For simplicity, our discussion is limited mainly to the case $n=2$.

Two main difficulties arise in the analysis of undercompressive shocks, that are not present in either the overcompressive or standard (Lax) shock case. The first is that the asymptotic state of a perturbed shock is no longer determined by the mass of the initial perturbation. This observation, made heuristically and numerically in [Zu-Pl-Ma], is verified analytically in Sect. 2.2. The second difficulty is that the characteristic speed in the wave field transverse to the shock is not bounded away from the shock speed as in the strictly hyperbolic case, but typically coincides with it at some point in space. Either one of these two features is enough to preclude the energy method for proving stability of shocks in systems of conservation laws [Mat, G.1, L.1, Sz-X].

In this method, the asymptotic state, consisting of a translated shock plus diffusion waves in the outgoing characteristic directions, can be determined from the perturbation mass by conservation, [L.1], because the number of parameters 
determining the asymptotic state is equal to the number of conservation laws. With the asymptotic state thus determined, the remainder has zero total mass. The equations for the remainder are then integrated and energy method applied for the stability analysis.

In the undercompressive case, the fact that the asymptotic shock location cannot typically be determined by the perturbation mass means that any analysis based on integrated variables allows the possibility of an order one error due to incorrect shock location. It follows that decay of the solution is not forced by the structure of the integrated equations, and the energy method described above must fail. Furthermore, even in the typical case that shock location can be determined by the perturbation mass, the amount of the diffusion waves still is not known a priori, and causes problems for the energy method approach.

The authors have previously shown how to deal with the second, essentially technical difficulty, in [L-Zu], where we analyzed the stability of a real Burgers shock within the larger system of the complex Burgers equation. In this case, the right eigenvectors of $f^{\prime}(\Phi( \pm \infty))$ associated with outgoing characteristic speeds are parallel, as a consequence of which the asymptotic location of the shock (though not the masses of outgoing diffusion waves) can still be determined by conservation of mass alone. The analysis involves Green's function methods and direct, pointwise estimates. Grosser estimates obtainable by energy or semigroup methods do not seem to suffice.

However, the more interesting, and characteristic aspect of the behavior of undercompressive shocks remains to be understood. An easy consequence of the fact that asymptotic shock location is generically not determined by the perturbation mass is that it is not given by any linear functional of the initial data (Sect. 2.3), but rather evolves nonlinearly. This is somewhat reminiscent of the case of asymptotic behavior for a hyperbolic system of conservation laws, where the right and left masses $p_{i}, q_{l}$ of different $N$-waves also give nonlinear time invariants of the solution [L.2]. But, in the case of an undercompressive wave, this extra structure is imposed purely by the dissipation term, and represents the inner structure of the shock. This we address in the present paper.

Plan of the paper. In Sect. 2, we develop a general approach to the stability of undercompressive shocks, based on the geometric property of the wave. We first examine the linearized equations around a general undercompressive shock wave solution $\Phi(x)$, taken without loss of generality to be stationary. By considering the dual equation, we determine all time-invariants of the linearized equations, in particular the asymptotic shift in the shock location. In the process, we elucidate the different character of Lax and undercompressive waves. In particular, we demonstrate that the undercompressive shock undergoes nonlinear movement under perturbation.

Using the time-invariants for the linearized perturbation variable $\bar{U}$, we are able to define a transformation $\tilde{U}=E(x) \bar{U}$ with the properties that:

(i) $\tilde{U}$ satisfies a system of conservation laws,

$$
\tilde{U}_{t}+(\tilde{f}(x) \tilde{U})_{x}=\tilde{U}_{x x},
$$

which are approximately decoupled.

(ii) The time-asymptotic state of $\tilde{U}$ (corresponding to asymptotic shift in shock location) is determined by $\int_{-\infty}^{+\infty} \tilde{U}_{1} d x$, where $\tilde{U}_{1}$ is the coordinate associated with the compressive characteristic field, a quantity which is exactly decoupled. 
Properties (i) and (ii) allow us to approximate the behavior of (2) by the behavior of its diagonal part, a system of decoupled scalar equations. The scalar equations have the important property that one of them governs the movement of the shock, while the other governs the movement of diffusion waves. This holds, of course, only for the linearized system.

This coordinate transformation is similar in principle to the diagonalization procedure used to analyze standard (Lax) shocks. However, the method of diagonalization takes into account parabolic as well as hyperbolic effects. The particular form of $E(x)$ is forced by the (necessary) requirements (i) and (ii).

At this point, we must deal with the nonlinear movement of the shock. Accordingly, we define an instantaneous location of the shock, $d(t)$, determined by the minimal requirement that at each time $t$ the solution must be linearly stable around $\Phi(x-d(t))$. This criterion is familiar from studies of traveling waves in more general settings, such as reaction-diffusion equations [Sat, Ga-J-Ka, Ka]. Also, note a related analysis in [G.3] treating the multidimensional stability of planar shock fronts for a scalar equation.

Combining this correction with the diagonalizing transformation for the linearized equations, we arrive at a final, nonlinear transformation,

$$
\tilde{U}(x, t)=E(x)[U(x+d(t), t)-\Phi(x, t)],
$$

which is convenient for analysis of nonlinear stability.

In Sect. 3, we apply the methods developed in Sect. 2 to an example system,

$$
\begin{cases}u_{t}+\frac{1}{2}\left(v^{2} u^{2}\right)_{x}+\varepsilon v_{x} & =u_{x x} \\ v_{t}+(u v)_{x} & =v_{x x}\end{cases}
$$

with shock

$$
\Phi(x)=\left(\begin{array}{c}
\varphi(x) \\
0
\end{array}\right)=\left(\begin{array}{c}
\tanh (x / 2) \\
0
\end{array}\right) .
$$

This is an $\varepsilon$-perturbation of the complex Burgers system treated in [L-Zu]. However, the property that the outgoing right eigenvectors are parallel has been broken, so that the undercompressive shock is now of generic, dynamic type. Our first calculation is to make explicit that, indeed, the analysis in [L-Zu] is not sufficient to treat this case.

After calculating the nonlinear transformation (2) explicitly, we find that the new variable, $\tilde{U}$, satisfies an equation which consists of the unperturbed complex Burgers equation plus source terms whose decay can be shown to be of sufficiently high rate. The analysis is completed using the pointwise methods from $[\mathrm{L}-\mathrm{Zu}]$, which apply directly to this case. For the transformed (perturbation) variable $\tilde{U}=\left(\begin{array}{c}\tilde{u} \\ \tilde{v}\end{array}\right)$, we obtain

Theorem 1 (Main Theorem). Let $C>1$ and $t_{0}>0$ be fixed, and let

$$
\begin{gathered}
\left|\tilde{u}_{0}(x)\right|,\left|\tilde{v}_{0}(x)\right| \leqq \delta e^{-x^{2} / 4 t_{0}}, \\
\int_{-\infty}^{\infty} \tilde{u}_{0}(x) d x=0 .
\end{gathered}
$$


Then, for $\delta$ sufficiently small, (1) has a global solution, for which $d(t)$ is well defined. Further, $d(t)=O(1) \varepsilon \delta^{2} e^{-b t}$ for $b>0$ and the transformed variable, $\tilde{U}$, satisfies

$$
\begin{aligned}
\tilde{u}(x, t)= & O(1) \delta\left[\left(t+t_{0}\right)^{-3 / 2} W_{C}\left(x, t+t_{0}\right)+K_{C}^{-2}\left(x, t+t_{0}\right)\right. \\
& \left.+K_{C}^{+^{2}}\left(x, t+t_{0}\right)+\left(t+t_{0}\right)^{-1 / 2} e^{-|x| / 2 C}\right] \\
\tilde{v}(x, t)= & O(1) \delta\left[K_{C}^{-}\left(x, t+t_{0}\right)+K_{C}^{+}\left(x, t+t_{0}\right)\right]
\end{aligned}
$$

where

$$
\begin{gathered}
W_{C} \triangleq \begin{array}{ll}
1 & -t \leqq x \leqq t \\
e^{\frac{-(x-t)^{2}}{4 C t}} & x \geqq t, \\
e^{\frac{-(x+t)^{2}}{4 C t}} & x \leqq-t,
\end{array} \\
K_{C}^{ \pm}(x, t) \triangleq K\left(\frac{x \mp t}{C}, t\right)=\frac{1}{\sqrt{4 \pi C t}} e^{-\frac{(1 \mp t)^{2}}{4 C t}},
\end{gathered}
$$

and $b, O(1)$ are constants depending only on $C, t_{0}$.

Theorem 2 ( $L^{p}$ Behavior). The variable $\tilde{u}$ converges to 0 in $L^{p}$ at the rate $t^{-1 / 2}$, while $\tilde{v}$ converges at rate $t^{-3 / 4+1 / 2 p}$ to a pair of heat kernels moving with speeds plus and minus 1, respectively.

This means that the original variable $U$ converges in $L^{p}$ at rate $\max \left\{t^{-1 / 2}\right.$, $\left.t^{-3 / 4+1 / 2 p}\right\}$ to the translated shock wave $\Phi(x-d(+\infty))$ plus two diffusion waves in the outgoing characteristic modes, precisely the asymptotic state we should expect by analogy with the standard (Lax) shock case (cf. [L.1]).

Finally, in Sect. 4, we discuss generalization and suggest future directions.

\section{Analytic Framework}

In this section, we show how to transform the perturbation equations of a general undercompressive shock into a standard, asymptotically diagonal form suitable for the analysis of stability. This transformation is different from the usual, hyperbolic diagonalization used to analyze perturbations of a classical (Lax) shock; in fact, the Jacobian of the inviscid flux may not even be diagonalizable everywhere along the shock profile. Rather, it depends in a fundamental way on the undercompressive nature of the shock.

1. Preliminaries. We restrict to the $2 \times 2$ case for simplicity. Let $\Phi(x-s t)$ be an undercompressive traveling wave solution of a viscous system of conservative laws,

$$
U_{t}+f(U)_{x}-\left(D(U) U_{x}\right)_{x}=0, \quad U \in R^{2},
$$

connecting end states

$$
\Phi_{ \pm \infty}=\lim _{x \rightarrow \pm \infty} \Phi(x) .
$$

Then, $f^{\prime}\left(\Phi_{ \pm \infty}\right)$ has real, distinct eigenvalues $\lambda_{l}\left(\Phi_{ \pm \infty}\right)$ satisfying the characteristic condition

$$
\lambda_{1}\left(\Phi_{-\infty}\right)>s>\lambda_{1}\left(\Phi_{+\infty}\right) ; \quad \lambda_{2}\left(\Phi_{-\infty}\right)<s<\lambda_{2}\left(\Phi_{+\infty}\right) .
$$


Let $l_{i}\left(\Phi_{ \pm \infty}\right), r_{i}\left(\Phi_{ \pm \infty}\right)$ denote the associated left and right eigenvectors. By the change of coordinates, $x^{\prime}=x-s t$, we can assume without loss of generality that $s=0$, i.e. $\Phi(x)$ is a standing wave solution. We wish to study the stability of $\Phi(x)$ with respect to a small $L^{1}$ perturbation $\bar{U}_{0}$.

For $\Phi$, we have in mind a weak $\left([\Phi] \equiv \Phi_{+\infty}-\Phi_{-\infty}\right.$ small) traveling wave bifurcating from some constant state solution $\Phi_{*}\left(x-s_{*} t\right) \equiv U_{*}$. By continuity, the base state $U_{*}$ must be nonstrictly hyperbolic, with $\lambda_{1}\left(U_{*}\right)=\lambda_{2}\left(U_{*}\right)=s_{*}$, and (by way of the Rankine-Hugoniot relation) $\lim _{[\Phi] \rightarrow 0} \frac{[\Phi]}{[[\Phi] \mid}$ must be an eigenvector of $f^{\prime}\left(U_{*}\right)$. This is a generalization of the classical weak shock wave bifurcating from a strictly hyperbolic base state.

Weak classical waves (cf. [Sm]) satisfy the Lax characteristic condition,

$$
\lambda_{1}\left(\Phi_{-\infty}\right)>s>\lambda_{1}\left(\Phi_{+\infty}\right) ; \quad \lambda_{2}\left(\Phi_{ \pm \infty}\right)>s \quad \text { or } \quad \lambda_{2}\left(\Phi_{ \pm \infty}\right)<s .
$$

By contrast, nonclassical waves bifurcating from a state of nonstrict hyperbolicity may be Lax type, undercompressive, or overcompressive. Overcompressive shocks satisfy

$$
\lambda_{1}\left(\Phi_{-\infty}\right)>s>\lambda_{1}\left(\Phi_{+\infty}\right) ; \quad \lambda_{2}\left(\Phi_{-\infty}\right)>s>\lambda_{2}\left(\Phi_{+\infty}\right) .
$$

Likewise, the characteristic structure is more complicated in the nonclassical case. Continuous functions $\lambda_{l}(U), l_{i}(U), r_{i}(U)$ typically cannot be defined in any neighborhood of the nonstrictly hyperbolic point $U_{*}$, or even along the profile $\Phi(x)$. Our labeling of characteristic speeds in (3) thus represents a considered choice, and is in fact our first step in uncovering a hidden characteristic structure imposed by the shock $\Phi$. Note that our labeling scheme does not follow the classical division into slow and fast waves $\left(\lambda_{1}<\lambda_{2}\right)$, but instead identifies a primary, compressive family $\left(\lambda_{1}\right)$ and a secondary, noncompressive family $\left(\lambda_{2}\right)$. This expresses more naturally the qualitative property of the shock.

2. Time Invariants of the Linearized Equations. We begin by studying the linearized perturbation equations. Linearizing (1) around $\Phi(x)$ gives a system

$$
\bar{U}_{t}=M \bar{U},
$$

which approximately describes the evolution of the perturbation $\bar{U}=U-\Phi(x)$, where

$$
M \bar{U}=\left(\left[D^{\prime}(\Phi) \Phi_{x}-f^{\prime}(\Phi)\right] \bar{U}\right)_{x}+\left(D(\Phi) \bar{U}_{x}\right)_{x}
$$

As with traveling waves of any type, e.g. dispersive, reaction-diffusion, etc., $\Phi(x)$ is at best orbitally stable under perturbation, since any translate $\Phi(x-d)$ is also a solution. Likewise, Eq. (2.7) is at most neutrally stable, since $\Phi^{\prime}(x)$ is always a stationary solution, associated with a zero-eigenvalue. $\Phi^{\prime}(x)$ is just the linearized version of a unit translation, and an asymptotic state $d \Phi^{\prime}(x)$ corresponds to a shift $d$ in the position of the shock.

A necessary condition for decay of $\bar{U}$ is that its asymptotic shift is zero. Accordingly, our first effort is to derive a linear decay criterion by calculating an expression for the shock shift in terms of the initial data, i.e. $d=d\left(\bar{U}_{0}\right)$. The function $d$ is 
clearly linear, and is expected to be continuous with respect to $\bar{U}_{0}$, that is, a linear functional on $L^{1}$. Since $(2.7)$ is autonomous, we have in fact that

$$
d=d(U(\cdot, t))
$$

for any fixed $t$, i.e. $d(\bar{U})$ is a time invariant of the solution $\bar{U}$.

More generally, we look for all $g(\bar{U})$ which are linear functionals on our solution space, $L^{1}$, and are time invariants of (2.7). Writing

$$
g(U)=\langle e(x), U\rangle=\int_{-\infty}^{\infty} e(x) U(x, t) d t
$$

with $e(x) \in L^{\infty}$, we find that time-invariance requires that

$$
0 \equiv \frac{d}{d t}\langle e, \bar{U}\rangle=\langle e, M \bar{U}\rangle=\left\langle M^{*} e, \bar{U}\right\rangle
$$

for all $\bar{U}$, i.e. $e(x)$ must satisfy the adjoint equation $M^{*} e=0$. Thus, the set of linear $L^{1}$-time-invariants of (2.7) corresponds exactly to the set of bounded solutions, $e(x)$, of

$$
M^{*} e=e_{x} f^{\prime}(\Phi)+e_{x x} D(\Phi)=0 .
$$

For classical, Lax shocks, the $L^{1}$ asymptotic state, hence all such time-invariants, can be determined from conservation of mass considerations (cf. [L.1]). That is, $d=e_{0} \int_{-\infty}^{\infty} u(x, t) d x=\left\langle e_{0}, \bar{U}\right\rangle$, for some constant vector $e_{0}$. However, this is not the case for undercompressive shocks, as was shown numerically in [Zu-Pl-Ma]. We now address this analytically.

Proposition 2.2.1. For $\Phi$ a Lax wave, the only bounded solutions of (2.11) are $e(x) \equiv$ constant. For $\Phi$ undercompressive, there exists a bounded nonconstant solution $e(x)$.

Proof. We recall that a Lax shock satisfies the characteristic condition (2.4), while an undercompressive shock satisfies (2.3). In each case, the equation

$$
M \bar{U}=0
$$

has but a single solution satisfying $\bar{U}( \pm \infty)=0$, namely $\Phi^{\prime}(x)$. For, integrating (2.12), we have

$$
D^{\prime}(\Phi) \Phi_{x}+f^{\prime}(\Phi) \bar{U}=D(\Phi) \bar{U}_{x},
$$

so that the frozen system at $x= \pm \infty$ is

$$
D\left(\Phi_{ \pm \infty}\right)^{-1} f^{\prime}\left(\Phi_{ \pm \infty}\right) \bar{U}=\bar{U}_{x} .
$$

Denote the eigenvalues and eigenvectors of $D\left(\Phi_{ \pm \infty}\right)^{-1} f^{\prime}\left(\Phi_{ \pm \infty}\right)$ by $\hat{\lambda}_{l}, \hat{l}_{l}$, and $\hat{r}_{i}$. Since $\operatorname{det}(D)>0$, and the orbit $\Phi_{x}$ exists, we have without loss of generality that $\hat{\lambda}_{i}$ also satisfy (2.4) or (2.3), respectively (otherwise, $-\hat{\lambda}_{i}$ must). But, these conditions correspond to repellor-saddle, saddle-attractor, and saddle-saddle connections, which are all unique.

Now, let $M^{*} e=0$, and set $W=e_{x}$. From (2.11), we have

$$
-W f^{\prime}(\Phi)=W_{x} D(\Phi) \text {. }
$$


Since the coefficients of (2.15) are asymptotically constant, the requirement $e(x) \in L^{\infty}$ is equivalent to $W(x) \in L^{1}$. In particular, $W(\mp \infty)=0$.

The eigenvalues and left eigenvectors of the frozen system

$$
W f^{\prime}\left(\Phi_{ \pm \infty}\right) D^{-1}\left(\Phi_{ \pm \infty}\right)=W_{x}
$$

are $-\hat{\lambda}_{l \pm \infty}$ and $\hat{l}_{ \pm \infty} D^{-1}\left(\Phi_{ \pm \infty}\right)$. We immediately see that $W(\mp \infty)=0$ is impossible in case (2.4), from consideration of the eigenvalues alone. For, this would correspond to an attractor-saddle or a saddle-repellor connection, which is impossible. We now consider case (2.3):

Lemma 2.1.2. $W(x) D(\Phi(x)) \bar{U}(x)$ is constant in $x$ for solutions $\bar{U}$ and $W$ of (2.12) and (2.15).

Proof.

$$
\begin{aligned}
\frac{d}{d x} W D(\Phi) \bar{U} & =W_{x} D \bar{U}+W D^{\prime} \Phi_{x} \bar{U}+W D \bar{U}_{x} \\
& =-W f^{\prime}(\Phi) \bar{U}+W D^{\prime} \Phi_{x} \bar{U}+W\left(D_{(\Phi)}^{\prime} \Phi_{x}+f^{\prime}(\Phi)\right) \bar{U} \\
& =0 .
\end{aligned}
$$

Note that $\bar{U}(\mp \infty)=0$ implies that

$$
\bar{U}(x) \sim e^{\left(\hat{\lambda}_{1 \mp \infty} x\right)} \hat{r}_{1 \mp \infty} \text { as } x \rightarrow \mp \infty,
$$

where $\hat{\lambda}_{1 \mp \infty}$ and $\hat{r}_{1 \mp \infty}$ are the stable eigenvalue and right eigenvector associated with $D\left(\Phi_{\mp \infty}\right)^{-1} f^{\prime}\left(\Phi_{\mp \infty}\right)$. Let $W(x)$ be a solution of (2.15) satisfying $W(0) D(\Phi(0) \bar{U}(0))=0$ and $W(0) \neq 0$.

By the lemma, $W D \bar{U} \equiv 0$, which implies that $W(\mp \infty)=0$ as well. For, otherwise,

$$
W(x) \sim e^{\left(-\hat{\hat{\lambda}}_{1 \mp \infty} x\right)} \hat{l}_{1 \mp \infty} D^{-1}\left(\Phi_{\mp \infty}\right)
$$

as $x \rightarrow \mp \infty$, since $-\hat{\lambda}_{1 \mp \infty}$ and $\hat{l}_{1 \mp \infty} D^{-1}\left(\Phi_{\mp \infty}\right)$ are the unstable eigenvalue and left eigenvector of $-f^{\prime}\left(\Phi_{\mp \infty}\right) D^{-1}\left(\Phi_{\mp \infty}\right)$. But, this would contradict the lemma, since then

$$
W D(\Phi) \bar{U} \sim \hat{l}_{1 \mp \infty} D^{-1}\left(\Phi_{\mp \infty}\right) D(\Phi) \hat{r}_{1 \mp \infty} \neq 0
$$

as $x \rightarrow \mp \infty$.

Therefore, there exists $W \neq 0$ solving (2.15), with $W( \pm \infty)=0$, which implies a nonconstant solution $e(x)$ of $M e=0$.

Remark. We note that $\bar{U}, W \in L^{1}$ play the role of dual variables. Lemma 2.12 and its use in constructing eigenvectors is reminiscent of the Evan's function methods used to study the spectrum of non-self-adjoint operators [A-Ga-J, E, Pe-W, K]. It would be interesting to clarify this connection further.

3. Linear Decay Criterion. The proof of Proposition 2.1.1 shows that the space of $L^{1}$ time-invariants is exactly three-dimensional for an undercompressive shock, and two dimensional for a Lax shock. The extra time-invariant in the undercompressive case agrees with our intuition that its asymptotic state should consist of a translated shock and two diffusion waves, rather than the translate and single diffusion wave of 
the Lax shock. Its existence would appear to be a necessary condition for stability of the shock.

We must determine the particular time-invariant $d(\bar{U})=\langle e(x), \bar{U}\rangle$ corresponding to shock shift. This is easily accomplished by a heuristic argument.

We can approximate the behavior of (2.6) near $X= \pm \infty$ by that of the frozen (constant coefficient) systems

$$
\bar{U}_{t}-\left(f^{\prime}\left(\Phi_{ \pm \infty}\right) \bar{U}\right)_{x}-D\left(\Phi_{ \pm \infty}\right) \bar{U}_{x x},
$$

and consider the asymptotic behavior resulting from initial data given as a point mass $\bar{U}_{0}(x)=\delta\left(x-x_{0}\right) v$, where $v$ is a vector and $x_{0}$ is near $\pm \infty$. Under reasonable assumptions, the asymptotic state is known (cf. [L-Ze]) to be

$$
\sum_{i} \ell_{i}\left(\Phi_{ \pm \infty}\right) v e^{-\frac{\left(x-i_{0}-\iota_{l}\left(\Phi_{ \pm \infty}\right) t\right)^{2}}{4 t}} r_{l}\left(\Phi_{ \pm \infty}\right) .
$$

If we choose $v=r_{2}\left(\Phi_{ \pm \infty}\right)$, then the asymptotic state is a single outgoing diffusion wave, which stays near $\pm \infty$. In this case, we may expect that the asymptotic state of (2.20) is very close to the asymptotic state of the linearized equation (2.6). Since there is no contribution to the shift and the diffusion wave on the opposite side, we conclude that $e(x)$ satisfies the boundary conditions

$$
e( \pm \infty) r_{2}\left(\Phi_{ \pm \infty}\right)=0 \text {. }
$$

In other words, $e( \pm \infty)$ are parallel to $\ell_{1}\left(\Phi_{ \pm \infty}\right)$.

Since $d\left(\Phi_{x}\right)=1$ by definition, $e(x)$ must further satisfy the normalization requirement

$$
\left\langle e(x), \Phi_{x}\right\rangle=1 .
$$

Together, these three requirements exactly specify $e(x)$ within the three dimensional space of time-invariants.

With this choice of $e(x)$, the criterion for linear decay is

$$
d(\bar{U})=\langle e(x), \bar{U}\rangle=0 .
$$

We comment that once the shock shift has been determined, the masses of the two outgoing diffusion waves can also be determined, by the two equations of conservation of mass. However, we do not need this information in our analysis.

Remark. 2.3.1. In the generic case that $e(x)$ is nonconstant, it is easy to see that the shock shift for the full, nonlinear equation is not given by any linear functional of the perturbation $\bar{U}=U-\Phi$.

For, such a functional would necessarily coincide with the linear shift functional, $d(\bar{U})=\langle e(x), \bar{U}\rangle$. But, then,

$$
\begin{aligned}
d / d t\langle e, \bar{U}\rangle & =\langle e, M \bar{U}\rangle+\left\langle e, S_{x}(\bar{U})\right\rangle \\
& =\left\langle e, S_{x}(\bar{U})\right\rangle \\
& =\int e(x) S_{x}(\bar{U}) d x \\
& =-\int e^{\prime}(x) S(\bar{U}) d x
\end{aligned}
$$

would vanish for all $\bar{U}$, implying that either $e(x) \equiv$ constant or else $S(\bar{U}) \equiv 0$. 
Thus, we already see that the location of an undercompressive shock must evolve nonlinearly, in contrast to the standard (Lax) shock case.

4. Diagonalizing Transformation. At this point, we restrict to the case $D(\Phi) \equiv I$, for simplicity. Let $E(x)=\left(\begin{array}{l}e_{1}(x) \\ e_{2}(x)\end{array}\right)$, satisfy the adjoint equation

$$
M^{*} E=E_{x} f^{\prime}(\Phi)+E_{x x}=0,
$$

and the boundary conditions

$$
e_{1}( \pm \infty) r_{2}\left(\Phi_{ \pm \infty}\right)=0 ; \quad e_{2}( \pm \infty) r_{1}\left(\Phi_{ \pm \infty}\right)=0 .
$$

We require $E(x)$ to be nonsingular for all $x$. This is a structural condition on $\Phi$ which must be verified on a case by case basis. However, it seems to hold quite generally for weak undercompressive shocks.

We introduce the transformation

$$
\tilde{U}=E(x) \bar{U} .
$$

Multiplying (2.6) by $E$, we find that

$$
\begin{aligned}
0 & =E\left(\bar{U}_{t}-M \bar{U}\right) \\
& =E \bar{U}_{t}+E\left(f^{\prime}(\Phi) \bar{U}_{x}\right)-E \bar{U}_{x x} \\
& =(E \bar{U})_{t}+\left(E f^{\prime}(\Phi) \bar{U}\right)_{x}-(E \bar{U})_{x x}-\left(M^{*} E\right) U,
\end{aligned}
$$

Since $\left(M^{*} E\right) U=0, \tilde{U}$ itself satisfies a conservation law,

$$
\tilde{U}_{t}-(\tilde{f}(x) \tilde{U})_{x}-\tilde{U}_{x x}=0
$$

where

$$
\tilde{f}(x)=E f^{\prime}(\Phi(x)) E^{-1}+2 E_{x} E^{-1} .
$$

By the boundary conditions (2.4.1), the convection matrix $\tilde{f}(x)$ satisfies

$$
\tilde{f}( \pm \infty)=\left(\begin{array}{cc}
\lambda_{1}\left(\Phi_{ \pm \infty}\right) & 0 \\
0 & \lambda_{2}\left(\Phi_{ \pm \infty}\right)
\end{array}\right),
$$

i.e. (2.29) asymptotically decouples. Thus, the coordinate transform (2.27) is somewhat analogous to the diagonalization usually performed in the analysis of stability of Lax shocks ([G.1, L.1, Sz-X]).

Further, since $e_{1}(x)$ is a multiple of the shift function $e(x)$, the linear decay criterion exactly decouples, becoming simply

$$
\int_{-\infty}^{\infty} \tilde{U}_{1} d x=0
$$

Properties (2.31-32) allow us to study the behavior of (2.29) by studying the behavior of its diagonal part, treating off-diagonal terms as higher order effects. In fact, they are necessary for this approach to succeed, (2.31) from obvious considerations and (2.32) because the asymptotic behavior of (2.29) and the diagonalized 
system must agree - it is well known that (2.32) determines the asymptotic state for a conservative scalar equation.

Condition (2.32) is unique to the undercompressive case. Such considerations are avoided in the standard (Lax) case by the use of integrated variables, for which the asymptotic state is built in.

Though our approach is similar in spirit to the standard approach used to study Lax shocks, note that the method of diagonalizing is quite different. In particular, the vectors $e_{i}(x)$ do not represent actual left eigenvectors of $f^{\prime}(\Phi(x))$, which in many cases do not exist. Rather, they represent effective modes of propagation resulting from the combined convective and dissipative effects of the shock profile $\Phi$. Their existence demonstrates that shock structure can induce hyperbolic behavior, even in regions of ellipticity.

5. Nonlinear Version. We now turn to the full, nonlinear system (1). As remarked in Sect. 3, the shock location evolves nonlinearly for the full equations. Accordingly, we denote the shock location at time $t$ by $d(t)$ and define the perturbation variable $\bar{U}$ as

$$
\bar{U}(x, t)=(U(x+d(t), t)-\Phi(x)) .
$$

Here, we have normalized by mapping the shock to the origin before computing the perturbation.

In terms of $\bar{U},(1)$ becomes

$$
\bar{U}_{t}-M \bar{U}=S_{x}+\dot{d}\left(\bar{U}_{x}+\Phi_{x}\right),
$$

where $M$ is the linearized operator (2.7) and

$$
S(\bar{U})=f(U)-f(\Phi)-f^{\prime}(\Phi)(U-\Phi)=0(1)|\bar{U}|^{2}
$$

is the nonlinear source.

Introducing $\tilde{U}=E(x) \bar{U}$, we have

$$
\tilde{U}_{t}+(\tilde{f}(x) \tilde{U})_{x}-\tilde{U}_{x x}=E S_{x}+\dot{d} E\left(\bar{U}_{x}+\Phi_{x}\right),
$$

or

$$
\tilde{U}_{t}+(\tilde{f}(x) \tilde{U})_{x}-\tilde{U}_{x x}=(\tilde{S}+\dot{d} \tilde{U})_{x}+\left(\tilde{N}+\dot{d}\left[E \Phi_{x}-E_{x} E^{-1} \tilde{U}\right]\right),
$$

where

$$
\tilde{S}=E S
$$

and

$$
\tilde{N}=E_{x} S \text {. }
$$

The location $d(t)$ can be determined implicitly by the requirement that the translated shock $\Phi(x-d(t))$ be at least linearly stable under the perturbation

$$
[U(x, t)-\Phi(x-d(t))]
$$

at each time $t$.

Equivalently, we require $\Phi(x)$ to be linearly stable under the perturbation $\tilde{U}=$ $U(x+d(t))-\Phi(x)$. In the $\tilde{U}$ coordinates, the linear decay criterion is simply the zero-mass requirement $\int_{-\infty}^{\infty} \tilde{U}(x, t){ }_{1} d x \equiv 0 \forall t$, by (2.32). 
Differentiating (2.32) with respect to $t$ gives

$$
\int_{-\infty}^{\infty} \tilde{U}_{1_{t}} d x=0
$$

Integrating (2.37) with respect to $x$ and applying (2.40) gives

$$
\int_{-\infty}^{\infty}\left(\tilde{N}_{1}+\dot{d}(t)\left[E \Phi_{x}-E_{x} E^{-1} \tilde{U}\right]_{1} d x=0\right.
$$

or

$$
\dot{d}(t)=\frac{\int_{-\infty}^{\infty} \tilde{N}_{1} d x}{\int_{-\infty}^{\infty}\left[E_{x} E^{-1} \tilde{U}-E \Phi_{x}\right]_{1} d x},
$$

completing the description of system (2.37).

The linear terms of the left-hand side are still asymptotically diagonal, revealing the stabilizing hyperbolic structure governing the behavior of a perturbation. Since we have mapped the shock location to $x \equiv 0$ in (2.37), the nonlinear source terms of the right-hand side presumably now represent secondary effects which can be estimated by the usual strategy of iteration. In Sect. 3, we demonstrate how this program can be carried out to show nonlinear stability around $\Phi(x-d(t))$.

\section{Application to an Example System}

We now demonstrate how the ideas of Sect. 2 can be used to obtain nonlinear stability, by their application to a particular system.

1. Perturbed Burgers Equation. We consider the system of conservation laws

$$
\begin{cases}u_{t}+\frac{1}{2}\left(v^{2}-u^{2}\right)_{x}+\varepsilon v_{x} & =u_{x x} \\ v_{t}+(u v)_{x} & =v_{x x}\end{cases}
$$

and the viscous shock wave solution

$$
\Phi(x)=\left(\begin{array}{c}
\varphi(x) \\
0
\end{array}\right)=\left(\begin{array}{c}
\tanh (x / 2) \\
0
\end{array}\right) .
$$

System (3.1) is a perturbation of the complex Burgers system $(\varepsilon=0)$ treated in $[\mathrm{L}-\mathrm{Zu}]$, and the shock $\Phi$ is a real Burgers shock, undercompressive in the surrounding system.

The inviscid system associated with (3.1) is of mixed type. The elliptic region is a ball of radius $\varepsilon / 4$, centered at $\left(\begin{array}{c}0 \\ -\varepsilon / 4\end{array}\right)$, and tangent to the shock profile $\Phi(x)$ at the origin. Thus, the origin is a point of nonstrict hyperbolicity, specifically, a parabolic degeneracy. $\Phi(x)$ is one of a family of undercompressive shocks bifurcating from the constant state solution $\Phi(x) \equiv 0$.

In complex form, with $z=u+i(v-\varepsilon / 4)$, (3.1) becomes

$$
z_{t}+\bar{z}_{x}^{2}-i \varepsilon / 2 z_{x}=z_{x x}
$$




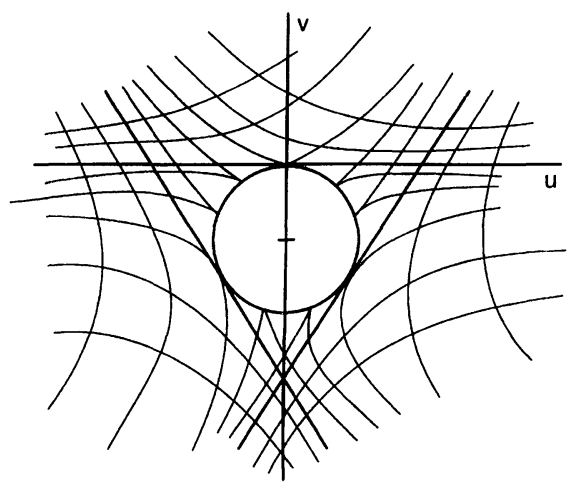

Fig. 3.1.

revealing threefold symmetry around the point $\left(\begin{array}{c}0 \\ -\varepsilon / 4\end{array}\right)$, via the invariance $z \rightarrow \omega z$, $\omega^{3}=1$. The line $\{v=0\}$ and its two symmetric counterparts are clearly invariant manifolds under the flow of both (3.1) and its associated inviscid system. These three invariant lines form an equilateral triangle circumscribing the elliptic region. The invariant manifolds of the inviscid flow are the wave curves, which we depict for reference in Fig. 3.1, superimposed on the elliptic region and the three invariant lines. These are integral manifolds of the eigenvectors of the flux jacobian, that is, of the local modes of propagation.

Linearizing $U=\left(\begin{array}{l}u \\ v\end{array}\right)$ about $\Phi$ gives

$$
\begin{cases}\bar{u}_{t}-(\varphi \bar{u}+\varepsilon \bar{v})_{x} & =\bar{u}_{x x} \\ \bar{v}_{t}+(\varphi \bar{v})_{x} & =\bar{v}_{x x} .\end{cases}
$$

Thus, along the shock profile $\Phi(x)$, the jacobian of the flux function of (3.1) is

$$
f^{\prime}(\Phi)=\left(\begin{array}{cc}
-\phi & \varepsilon \\
0 & \phi
\end{array}\right) .
$$

The associated eigenvalues are

$$
\lambda_{1}=-\phi ; \quad \lambda_{2}=\phi
$$

and the left and right eigenvectors are

$$
l_{1}=(\phi, \varepsilon / 2) ; \quad l_{2}=(0,1)
$$

and

$$
r_{1}=\left(\begin{array}{l}
1 \\
0
\end{array}\right) ; \quad r_{2}=\left(\begin{array}{c}
-\varepsilon / 2 \\
\phi
\end{array}\right)
$$

Note that for $\varepsilon>0$, the outgoing modes $r_{2}\left(\Phi_{ \pm \infty}\right)$ are not parallel, so the system is of generic (dynamic) type, in contrast to the degenerate (fixed shock) complex 
Burgers system $(\varepsilon=0)$ analyzed previously. We shall see that the behavior and the analysis are quite different as a result.

2. The case $\varepsilon=0$. When $\varepsilon=0$, (3.4) takes the form

$$
\left\{\begin{array}{l}
L_{1} \bar{u}=\bar{u}_{t}-(\varphi \bar{u})_{x}-\bar{u}_{x x}=0 \\
L_{2} \bar{v}=\bar{v}_{t}+(\varphi \bar{v})_{x}-\bar{v}_{x x}=0
\end{array}\right.
$$

This system was analyzed completely in $[\mathrm{L}-\mathrm{Zu}]$. We state the relevant results here without proof:

First, if $\int \bar{u}_{0} d x=0$, then the solution of (3.9) is given by

$$
\left\{\begin{array}{l}
\bar{u}(x, t)=\int_{-\infty}^{\infty} \overline{\mathscr{U}}(y, 0) G_{1}(x t ; y) d y \\
\bar{v}(x, t)=\int_{-\infty}^{\infty} \bar{v}(y, 0) G_{2}(x t ; y) d y
\end{array}\right.
$$

where

$$
\begin{aligned}
\overline{\mathscr{U}}(x, 0) \stackrel{\Delta}{=} & \int_{-\infty}^{x} \bar{u}(y, 0) d y, \\
G_{1}(x, t ; y)= & {\left[\alpha^{-}(x) K_{x}^{+}(x-y, t)+\alpha^{+}(x) K_{x}^{-}(x-y, t)\right] } \\
& -\left[\alpha^{\prime}(x) K^{+}(x-y, t)+\alpha^{\prime}(x) K^{-}(x-y, t)\right], \\
G_{2}(x, t ; y)= & \alpha^{-}(y) K^{-}(x-y, t)+\alpha^{+}(y) K^{+}(x-y, t),
\end{aligned}
$$

and

$$
\alpha^{\mp}(x)=\frac{e^{\mp x / 2}}{e^{x / 2}+e^{-x / 2}}, K^{ \pm}(x, t)=K(x \mp t, t)=\frac{1}{\sqrt{4 \pi t}} \frac{e^{-(x \mp t)^{2}}}{4 t} ; \quad \alpha^{\prime}(x) \stackrel{\Delta}{=} \alpha^{+^{\prime}}(x) .
$$

An immediate consequence is that, for initial data of compact support, $\bar{u}$ decays exponentially and $\bar{v}$ decays as $t^{-1 / 2}$.

Now, define

$$
W_{C} \triangleq \begin{cases}1 & -t \leqq x \leqq t \\ e^{\frac{-(x-t)^{2}}{4 C t}} & x \geqq t, \\ e^{\frac{-(x+t)^{2}}{4 C t}} & x \leqq-t,\end{cases}
$$

and

$$
K_{C}^{ \pm}(x, t) \stackrel{\Delta}{=} K\left(\frac{x \mp t}{C}, t\right)=\frac{1}{\sqrt{4 \pi C t}} e^{-\frac{(x \mp t)^{2}}{4 C t}} .
$$

Proposition 3.1.1. Let $L_{1} u=\left(S_{1}\right)_{x}, u(x, 0) \equiv 0$, and $C>1$.

(i) If

$$
|S|=O(1)\left[(t+1)^{-r} W_{C}(x, t+1)+(t+1)^{-q} e^{-|x| / 2 C}\right],
$$


and

$$
\left|S_{x}\right|=O(1) t^{-1 / 2}\left[(t+1)^{-r+1 / 2} W_{C}(x, t+1)+(t+1)^{-q+1 / 2} e^{-|x| / 2 C}\right],
$$

then

$$
|u|,\left|u_{x}\right|=O(1)\left[(t+1)^{-r+1 / 2} W_{C}(x, t+1)+(t+1)^{-\min \{q, r-1\}} e^{-|x| / 2 C}\right] .
$$

(ii) If

$$
|S|=O(1)(t+1)^{-p}\left[K_{C}^{-}(x, t+1)^{2}+K_{C}^{+}(x, t+1)^{2}\right]
$$

and

$$
\left|S_{x}\right|=O(1) t^{-1 / 2}(t+1)^{-p}\left[K_{C}^{-}(x, t+1)^{2}+K_{C}^{+}(x, t+1)^{2}\right],
$$

then

$$
|u|,\left|u_{x}\right|=O(1)\left[(t+1)^{-p-1} W_{C}(x, t+1)+(t+1)^{-p-1 / 2} e^{-|x| / 2 C}\right] .
$$

Proposition 3.1.2. Let $L_{2} v=\left(S_{2}\right)_{x}, v(x, 0) \equiv 0$, and $C>1$. If

(i)

$$
|S|=O(1)\left[(t+1)^{-1 / 2} e^{-|x| / 2 C}+(t+1)^{-1}\right]\left[K_{C}^{-}(x, t+1)+K_{C}^{+}(x, t+1)\right]
$$

and

$$
\left|S_{x}\right|=O(1) t^{-1 / 2}\left[K_{C}^{-}(x, t+1)+K_{C}^{+}(x, t+1)\right],
$$

or

(ii)

$$
|S|=O(1) t^{-1 / 2}\left[K_{C}^{-}(x, t+1)+K_{C}^{+}(x, t+1)\right]
$$

and

$$
\left|S_{x}\right|=O(1) t^{-1}\left[K_{C}^{-}(x, t+1)+K_{C}^{+}(x, t+1)\right],
$$

then

$$
|v|=O(1)\left[K_{C}^{-}(x, t+1)+K_{C}^{+}(x, t+1)\right]
$$

and

$$
\left|v_{x}\right|=O(1)(t+1)^{-1 / 2}\left[K_{C}^{-}(x, t+1)+K_{C}^{+}(x, t+1)\right] .
$$

Remark 3.3.1. From (3.10), it follows that $v=O(1)\left[K_{C}^{+}+K_{C}^{-}\right]$for $\varepsilon=0$ and initial data of compact support. If we attempt to treat $\varepsilon \bar{v}_{x}$ as a source term (i.e. negligible perturbation) in (3.4), we find by Proposition 3.1.2 (ii) that the result is $O(1) \varepsilon[(t+$ $\left.1)^{-1 / 2} W_{c}+e^{-|x| / 2 C}\right]$. This corresponds to an $O(1)$ shift of the shock, and precludes the possibility of a nonlinear analysis by perturbation techniques.

This is consistent with the observation that the shock location evolves nonlinearly, Remark 2.3.1. Clearly, the principal behavior of (3.4) is not given by (3.9) when $\varepsilon \neq 0$.

3. Linearized Stability. We now apply the ideas of Sect. 2, setting

$$
\tilde{U}(x, t)=E(x) \bar{U}(x, t),
$$

where $\bar{U}$ satisfies the linearized equations (3.4) and $E(x)$ satisfies conditions $(2.25-26)$. 
In the present case, the straight-line profile of $\Phi(x)$ makes $E(x)=\left(\begin{array}{l}e_{1}(x) \\ e_{2}(x)\end{array}\right)$ easy to compute. From $\Phi(x)=\left(\begin{array}{c}\varphi(x) \\ 0\end{array}\right)$ and the relation $\left(e_{l}\right)_{x} \Phi_{x} \equiv 0$ given in 3 , Lemma 2.1.2, we have

$$
\left(e_{l}\right)_{x}=W(x)=(0, w(x)) .
$$

Since $(0,1)$ is a left eigenvector of $f^{\prime}(\Phi(x))$ for all $x$, (2.25) becomes $w_{x}=$ $-\phi(x) w$, or

$$
w(x)=e^{\int_{0}^{x}-\varphi(x) d x}=O(1) e^{-|x|} .
$$

Condition (2.26) is $e_{i}( \pm \infty) r_{i}\left(\Phi_{ \pm \infty}\right)=0$. Combining (3.8) and (3.18), we obtain the solution

$$
E(x)=\left(\begin{array}{l}
e_{1}(x) \\
e_{2}(x)
\end{array}\right)=\left(\begin{array}{cc}
1 & \varepsilon \mathscr{W}(x) \\
0 & 1
\end{array}\right),
$$

where $\mathscr{W}( \pm \infty)=\mp 1 / 2$ and $\mathscr{W}_{x}=w(x)$.

We note that $e_{1}(x)$ is not constant, as a consequence of the fact that the eigenvectors $r_{1 \pm \infty}$ are not parallel. This implies that the shock is of generic (dynamic) type (Remark 2.3.1), in agreement with the calculation in Remark 3.3.1.

Computing

$$
E^{-1}=\left(\begin{array}{cc}
1 & -\varepsilon \mathscr{W}(x) \\
0 & 1
\end{array}\right)
$$

and

$$
E_{x}=\left(\begin{array}{cc}
0 & \varepsilon w(x) \\
0 & 0
\end{array}\right)
$$

and using (2.29-30), we find that the equations for $\tilde{U}=E(x) \bar{U}$ become

$$
\tilde{U}_{t}=(\tilde{f} \tilde{U})_{x}=\tilde{U}_{x x}
$$

where $\tilde{f}=\left(\begin{array}{cc}-\phi & \varepsilon a(x) \\ 0 & \phi\end{array}\right)$. Since $\tilde{f}$ is diagonal at $x= \pm \infty$, and all factors of $\tilde{f}$ decay as $w(x)=O(1) \varepsilon e^{-|x|}$ to their states at $\pm \infty$, we have $a(x)=O(1) e^{-|x|}$.

Thus, we can rewrite (3.20) as

$$
\left\{\begin{array}{l}
L_{1} \tilde{u}=\tilde{u}_{t}+(-\varphi(x) \tilde{u})_{x}-\tilde{u}_{x x}=\left(\varepsilon a_{1}(x) \tilde{v}\right)_{x} \\
L_{2} \tilde{v}=\tilde{v}_{t}+(-\varphi(x) \tilde{v})_{x}-\tilde{v}_{x x}=0
\end{array}\right.
$$

where $L_{1}$ and $L_{2}$ are as in (3.9) and $a_{1}(x)=O(1) e^{-|x|}$.

Now the linear source term, $\left(\varepsilon a_{1}(x) \tilde{v}\right)_{x}$, has form

$$
\varepsilon e^{-|x|} \tilde{v}=O(1) \varepsilon e^{-|x| / 2 C} e^{-|x| / 2 C}\left[K_{C}^{+}+K_{C}^{-}\right]=O(1) \varepsilon e^{-b t} e^{-|x| / 2 C},
$$

for $b>0(C>1)$. By Proposition 3.1.1 (i), this source gives contribution

$$
O(1) \varepsilon e^{-b t} e^{-|x| / 2 C}
$$


a negligible term. Therefore, linearized stability is trivial in the new coordinates, and the condition for stability is

$$
\int_{0}^{\infty} \tilde{u}_{0} d x=0, \quad \text { or } \int_{-\infty}^{\infty} e_{1}(x) \bar{U}_{0}(x) d x=0
$$

as predicted in Sect. 2 .

4. Nonlinear Stability. Following Sect. 2.5, we study the nonlinear stability of $\Phi$ by setting

$$
\tilde{U}(x, t)=E(x)[U(x+d(t), t)-\Phi(x)],
$$

where $U=\left(\begin{array}{l}u \\ v\end{array}\right)$ satisfies the full, nonlinear equations (3.1), $E(x)$ is as in (2.22), and $d(t)$ is the instantaneous shock location, as defined in Sect. 2.

By (2.37) and (2.42), $\tilde{U}$ and $d$ together satisfy the system of equations

$$
\left\{\begin{array}{l}
\tilde{U}_{t}+(\tilde{f} \tilde{U})_{x}-\tilde{U}_{x x}=(\tilde{S}+\dot{d} \tilde{U})_{x}+\left(\tilde{N}+\dot{d}\left[E \Phi_{x}-E_{x} E^{-1} \tilde{U}\right]\right) \\
\dot{d}(t)=\frac{\int_{-\infty}^{\infty} \tilde{N}_{1} d x}{\int_{-\infty}^{\infty}\left[E_{x} E^{-1} \tilde{U}-E \Phi_{x}\right]_{1} d x}
\end{array} .\right.
$$

Using (2.35), (2.38-39), and (3.19-21), and $\tilde{v}=\bar{v}$, we have

$$
\begin{gathered}
S=\left(\begin{array}{c}
1 / 2 \bar{u}^{2}-1 / 2 \bar{v}^{2} \\
-\bar{u} \bar{v}
\end{array}\right), \\
\tilde{S}=E S=\left(\begin{array}{c}
b_{1}(x) \tilde{u}^{2}+b_{2}(x) \tilde{u} \tilde{v}+b_{3}(x) \tilde{v}^{2} \\
\left(\tilde{u}+b_{4}(x) \tilde{v}\right) \tilde{v}
\end{array}\right), \\
\tilde{N}=E_{x} S=\left(\begin{array}{c}
\varepsilon a_{2}(x)\left(\tilde{u}+a_{4}(x) \tilde{v}\right) \tilde{v} \\
0
\end{array}\right),
\end{gathered}
$$

and

$$
\left[E \Phi_{x}-E_{x} E^{-1} \tilde{U}\right]=\left[\Phi_{x}+E_{x} \tilde{U}\right]=\left(\begin{array}{c}
\frac{\varepsilon}{2} a_{4}(x) \tilde{v}+\varphi_{x} \\
0
\end{array}\right),
$$

where $b_{i}=0(1), b_{l_{x}}=0(1) O(1) e^{-|x|}$, and $a_{l},\left(a_{l}\right)_{x}=O(1) e^{-|x|}$.

Defining

$$
\tilde{T}(x, t)=\int_{-\infty}^{x}\left[\tilde{N}-\dot{d}(t)\left(\Phi_{x}+E_{x} \tilde{U}\right)\right] d x
$$

and using (3.23), (3.30), we rewrite (3.26) as

$$
\left\{\begin{array}{l}
L \tilde{U}=\left(\begin{array}{c}
\varepsilon a_{1}(x) \tilde{v} \\
0
\end{array}\right)_{x}+(\tilde{S}+\tilde{T}+\dot{d}(t) \tilde{U})_{x} \\
\dot{d}(t)=\frac{\int \bar{N}_{1} d x}{\int\left[\frac{\varepsilon}{2} a_{4}(x) \tilde{v}+\varphi_{x}\right] d x}
\end{array}\right.
$$


where $L=\left(\begin{array}{l}L_{1} \\ L_{2}\end{array}\right)$ is as in (3.9). Note that $\tilde{T}( \pm \infty)=0$, by (2.41) and (3.29-30), so that the equation for $\tilde{U}$ is in conservation form.

Proposition 3.4.1 ( $L^{1}$ bounded stability). Let $C>1$ and let $\left|\tilde{u}_{0}(x)\right|,\left|\tilde{v}_{0}(x)\right| \leqq$ $\delta e^{-x^{2} / 4}$ and $\int_{-\infty}^{\infty} \tilde{u}_{0}(x) d x=0$.

Then, for $\varepsilon, \delta$ sufficiently small, (3.32) has a global solution, which satisfies

$$
\dot{d}(t)=O(1) \varepsilon \delta^{2} e^{-b t},
$$

for some $b>0$, and

$$
\begin{aligned}
\tilde{u}(x, t) & =O(1) \delta\left[(t+1)^{-1} W_{C}(x, t+1)+(t+1)^{-1 / 2} e^{-|x| / 2 C}\right], \\
\tilde{u}_{x}(x, t) & =O(1) \delta\left[t^{-1 / 2}(t+1)^{-1 / 2} W_{C}(x, t+1)+(t+1)^{-1 / 2} e^{-|x| / 2 C}\right], \\
\tilde{v}(x, t) & =O(1) \delta\left[K_{C}^{-}(x, t+1)+K_{C}^{+}(x, t+1)\right], \\
\tilde{v}_{x}(x, t) & =O(1) \delta t^{-1 / 2}\left[K_{C}^{-}(x, t+1)+K_{C}^{+}(x, t+1)\right],
\end{aligned}
$$

where

$$
\begin{gathered}
W_{C} \triangleq \begin{cases}1 & -t \leqq x \leqq t \\
e^{\frac{-(x-t)^{2}}{4 C t}} & x \geqq t, \\
e^{\frac{-(x+t)^{2}}{4 C t}} & x \leqq-t,\end{cases} \\
K_{C}^{ \pm}(x, t) \triangleq\left(\frac{x \mp t}{C}, t\right)=\frac{1}{\sqrt{4 \pi C t}} e^{\frac{-(x \mp t)^{2}}{4 C t}},
\end{gathered}
$$

and $O(1)$ is a constant depending only on $C$.

Proof. The proof is similar to that of Proposition 4.1 in $[\mathrm{L}-\mathrm{Zu}]$, though slightly more complicated.We proceed by iteration. Let $\left\{\tilde{U}_{k}, d_{k}\right\}$ be the sequence defined by

$$
\tilde{U}_{0} \equiv 0 ; \quad d_{0} \equiv 0
$$

and

$$
\begin{aligned}
L\left(\tilde{U}_{k+1}\right) & =\left(\begin{array}{c}
\varepsilon a_{1}(x) \tilde{v}_{k} \\
0
\end{array}\right)_{x}+\left[\tilde{S}\left(\tilde{U}_{k}\right)+\tilde{T}\left(\tilde{U}_{k}, \dot{d}_{k+1}\right)+\dot{d}_{k+1}(t) \tilde{U}_{k}\right]_{x}, \\
\tilde{U}_{k+1}(x, 0) & =\tilde{U}^{0}(x) \\
\dot{d}_{k+1}(t) & =\frac{\int \tilde{N}\left(\tilde{U}_{k}\right)_{1} d x}{\int\left[\frac{\varepsilon}{2} a_{4}(x) \tilde{v}_{k}+\varphi_{x}\right] d x},
\end{aligned}
$$

where $\tilde{S}, \tilde{N}$, and $\tilde{T}$ are as in (3.28-29), (3.31), and $\tilde{U}^{0}=\left(\begin{array}{l}\tilde{u}_{0} \\ \tilde{v}_{0}\end{array}\right)$ denotes the initial data for Eq. (3.4.2).

Short time existence theory (cf. [H-Sm]) guarantees that the iterates $\left\{\tilde{U}_{k}, d_{k}\right\}$ converge to a global solution of (4.1), provided that $\left|\tilde{U}_{k}\right|$ and $\left|\dot{d}_{k}(t)\right|$ are uniformly bounded. 
Lemma 4.1.a (A Priori Estimate). The sequence $\left\{\tilde{U}_{k}, d_{k}\right\}$ satisfies

$$
\begin{aligned}
\tilde{u}_{k}(x, t) & \leqq \delta_{k}\left[(t+1)^{-1} W_{C}(x, t+1)+(t+1)^{-1 / 2} e^{-|x| / 2 C}\right], \\
\tilde{u}_{k_{x}(x, t)} & \leqq \delta_{k}\left[t^{-1 / 2}(t+1)^{-1 / 2} W_{C}(x, t+1)+(t+1)^{-1 / 2} e^{-|x| / 2 C}\right], \\
\tilde{v}_{k}(x, t) & \leqq \delta_{k}\left[K_{C}^{-}(x, t+1)+K_{C}^{+}(x, t+1)\right], \\
\tilde{v}_{k_{x}(x, t)} & \leqq \delta_{k}(t)^{-1 / 2}\left[K_{C}^{-}(x, t+1)+K_{C}^{+}(x, t+1)\right], \\
\left|\dot{d}_{k}(t)\right| & \leqq 0(1) \varepsilon \delta_{k-1}^{2} e^{-b t},
\end{aligned}
$$

with $b>0$, where $\delta_{0}=0$ and, so long as $\varepsilon \delta_{k}$ is sufficiently small,

$$
\delta_{k+1}=O(1)\left(\delta+\delta_{k}^{2}+\varepsilon \delta_{k}\right) .
$$

The constants $O(1)$ depend only on $C$.

Proof. We proceed by induction, noting that the assertion holds trivially for $k=0$.

We can write $\tilde{U}_{k}=\hat{U}_{k}+\hat{U}_{k}$, where

$$
L\left(\hat{U}_{k+1}\right)=0 ; \quad \hat{U}_{k+1}(x, 0)=\tilde{U}^{0}(x)
$$

and

$$
\begin{aligned}
L\left(\hat{U}_{k+1}\right) & =\left(\begin{array}{c}
\varepsilon a_{1}(x) \tilde{v}_{k} \\
0
\end{array}\right)_{x}+\left[\tilde{S}\left(\tilde{U}_{k}\right)+\tilde{T}\left(\tilde{U}_{k}, \dot{d}_{k+1}\right)+\dot{d}_{k+1}(t) \tilde{U}_{k}\right]_{x} \\
\dot{d}_{k+1}(t) & =\frac{\int \tilde{N}\left(\tilde{U}_{k}\right)_{1} d x}{\int\left[\frac{\varepsilon}{2} a_{4}(x) \tilde{v}_{k}+\varphi_{x}\right] d x}, \\
\hat{U}_{k+1}(x, 0) & =0 .
\end{aligned}
$$

We first estimate $\dot{d}(t)$. We have

$$
\begin{gathered}
\left.\int_{-\infty}^{\infty} \varphi_{x} d x=\varphi\right]_{-\infty}^{\infty}=2, \\
\int \frac{\varepsilon}{2} a_{4}(x) \tilde{v}_{k} d x=0(1) \varepsilon \delta_{k} \int a_{4}(x) d x=0(1) \varepsilon \delta_{k}
\end{gathered}
$$

and

$$
\begin{aligned}
\int_{-\infty}^{\infty} \tilde{N}\left(\tilde{U}_{k}\right)_{1} d x & =0(1) \varepsilon \int e^{-|x|}(\tilde{u}+b(x) \tilde{v}) \tilde{v} d x \\
& =0(1) \varepsilon \delta_{k}^{2}\left(e^{-|x| / 2} K_{C}^{ \pm}(x, t+1)\right) \int e^{-|x| / 2} d x \\
& =0(1) \varepsilon \delta_{k}^{2} e^{-b t},
\end{aligned}
$$

for some $b>0$. Thus, for $\varepsilon \delta_{k}$ sufficiently small,

$$
\dot{d}_{k+1}(t)=\frac{0(1) \varepsilon \delta_{k}^{2} e^{-b t}}{\left(2+0(1) \varepsilon \delta_{k}\right)}=0(1) \varepsilon \delta_{k}^{2} e^{-b t},
$$

as claimed. 
We can now estimate $\tilde{T}$. We have

$$
\tilde{N}\left(\tilde{U}_{k}\right)=0(1) \varepsilon \delta_{k}^{2}\left(\begin{array}{c}
e^{-b t} e^{-|x| / 2} \\
0
\end{array}\right)
$$

and

$$
\dot{d}_{k+1}(t)\left(\Phi_{x}+E_{x} \tilde{U}_{k}\right)=0(1) \varepsilon \delta_{k}^{2}\left(\begin{array}{c}
e^{-b t} e^{-|x| / 2} \\
0
\end{array}\right) .
$$

For $x<0$, this implies that

$$
\begin{aligned}
\tilde{T}\left(\tilde{U}_{k}, \dot{d}_{k+1}\right) & =\int_{-\infty}^{x}\left[\tilde{N}\left(\tilde{U}_{k}\right)-\dot{d}(t)\left(\Phi_{x}+E_{x} \tilde{U}_{k}\right)\right] d x \\
& =0(1) \varepsilon \delta_{k}^{2} e^{-b t} \int_{-\infty}^{x} e^{-|x| / 2} d x \\
& =0(1) \varepsilon \delta_{k}^{2} e^{-b t} e^{-|x| / 2} .
\end{aligned}
$$

Further, $\tilde{T}(+\infty)=\int_{-\infty}^{+\infty}[\cdots] d x=0$, as noted after (3.4.2). Thus, for $x>0$, we can use the alternate representation

obtaining the bound

$$
\tilde{T}=\int_{x}^{\infty}\left[\tilde{N}-\dot{d}(t)\left[\Phi_{x}+E_{x} \tilde{U}\right] d x,\right.
$$

$$
\tilde{T}\left(\tilde{U}_{k}, \dot{d}_{k+1}\right)=0(1) \varepsilon \delta_{k}^{2}\left(e^{-b t} e^{-|x| / 2}\right)
$$

for all $x$. That $\left(\tilde{T}_{x}\right)=0(1) \varepsilon \delta_{k}^{2} e^{-b t} e^{-|x| / 2}$ follows immediately from (3.47-48).

The estimates of $\dot{d}$ and $\tilde{T}$ are the crucial new features in this proof. The remainder of the argument follows essentially as in Lemma 4.1.a in [L-Zu]:

We have

$$
L_{1}\left(\hat{\hat{u}}_{k+1}\right)=\left(\mathscr{S}_{1}\right)_{x}
$$

and

$$
L_{1}\left(\hat{\hat{v}}_{k+1}\right)=\left(\mathscr{S}_{2}\right)_{x}
$$

where

$$
\mathscr{S}_{1}=\left(\varepsilon a_{1}(x) \tilde{v}+\tilde{S}_{1}+\tilde{T}_{1}+\dot{d}(t) \tilde{u}\right)
$$

and

$$
\mathscr{S}_{2}=\left(\tilde{S}_{2}+\tilde{T}_{2}+\dot{d}(t) \tilde{v}\right) .
$$

From (3.28), we recall that

$$
\tilde{S}_{1}=O(1)\left(\left|\tilde{u}_{k}\right|^{2}+\left|\tilde{v}_{k}\right|^{2}\right)
$$

and

$$
\tilde{S}_{2}=\tilde{v}_{k} \tilde{u}_{k}+b(x) \tilde{v}_{k}^{2},
$$

where $b_{x}=O(1) e^{-|x|}$. 
Thus,

$$
\begin{aligned}
\tilde{S}_{1}= & O(1) \delta_{k}^{2}\left[(t+1)^{-2} W_{C}(x, t+1)+(t+1)^{-1} e^{-|x| / 2 C}\right. \\
& \left.+K_{C}^{+}(x, t+1)^{2}+K_{C}^{+}(x, t+1)^{2}\right] ; \\
\tilde{S}_{1_{1}}= & O(1) \delta_{k}^{2}\left[t^{-1 / 2}(t+1)^{-3 / 2} W_{C}(x, t+1)+(t+1)^{-1} e^{-|x| / 2 C}\right. \\
& \left.+K_{C}^{+}(x, t+1)^{2}+K_{C}^{+}(x, t+1)^{2}\right] .
\end{aligned}
$$

Combining (3.57), (3.45), (3.50), and the estimates $\varepsilon a_{1}(x) \tilde{v}_{k}=O(1) \varepsilon \delta_{k} e^{-b t} e^{-|x| / 2}$ and $\varepsilon\left(a_{1}(x) \tilde{v}_{k}\right)_{x}=O(1) \varepsilon \delta_{k} e^{-b t} e^{-|x| / 2}$, we have

$$
\begin{aligned}
\mathscr{S}_{1}= & O(1)\left(\delta_{k}^{2}+\varepsilon \delta_{k}+\varepsilon \delta_{k}^{2}\right)\left[(t+1)^{-2} W_{C}(x, t+1)+(t+1)^{-1} e^{-|x| / 2 C}\right. \\
& \left.+K_{C}^{+}(x, t+1)^{2}+K_{C}^{+}(x, t+1)^{2}\right] ; \\
\mathscr{S}_{1_{x}}= & O(1)\left(\delta_{k}^{2}+\varepsilon \delta_{k}+\varepsilon \delta_{k}^{2}\right)\left[t^{-1 / 2}(t+1)^{-3 / 2} W_{C}(x, t+1)+(t+1)^{-1} e^{-|x| / 2 C}\right. \\
& \left.+K_{C}^{+}(x, t+1)^{2}+K_{C}^{+}(x, t+1)^{2}\right] .
\end{aligned}
$$

Thus, applying Proposition 3.1.1 and linearity, we find that

$$
\begin{aligned}
\hat{\hat{u}}_{k+1}= & O(1)\left(\delta_{k}^{2}+\varepsilon \delta_{k}+\varepsilon \delta_{k}^{2}\right)\left[(t+1)^{-1} W_{C}(x, t+1)+(t+1)^{-1 / 2} e^{-|x| / 2 C}\right], \\
\hat{\hat{u}}_{k+1_{\lambda}}= & O(1)\left(\delta_{k}^{2}+\varepsilon \delta_{k}+\varepsilon \delta_{k}^{2}\right)\left[t^{-1 / 2}(t+1)^{-1 / 2} W_{C}(x, t+1)\right. \\
& \left.+(t+1)^{-1 / 2} e^{-|x| / 2 C}\right]
\end{aligned}
$$

as claimed.

The bounds on $\hat{\hat{v}}_{k+1}$ and $\hat{\hat{v}}_{k+1_{x}}$ follow similarly, from Proposition 3.1.2. The only subtlety is to estimate the contribution of the source term $b(x) \tilde{v}_{k}^{2}$ separately from the others, using 3.1.2(ii) and the estimates

and

$$
b \tilde{v}_{k}^{2}=O(1)(t+1)^{-1 / 2} K_{C}^{ \pm}
$$

$$
\begin{aligned}
\left(b \tilde{v}_{k}^{2}\right)_{x} & =\left(b_{x} \tilde{v}_{k}\right) \tilde{v}_{k}+b \tilde{v}_{k} \tilde{v}_{k_{x}} \\
& =O(1) e^{-b t} e^{-|x| / 2} K_{C}^{ \pm}+O(1) t^{-1 / 2}(t+1)^{-1 / 2} K_{C}^{ \pm}
\end{aligned}
$$

Here, we have used $b=O(1), b_{x}=O(1) e^{-|x|}$.

As in $[\mathrm{L}-\mathrm{Zu}]$, we can obtain similar bounds on the linear parts, $\hat{u}_{k+1}, \hat{u}_{k+1_{x}}, \hat{v}_{k+1}$, and $\hat{v}_{k+1_{x}}$, by direct calculation of the convolution integrals in (3.10). Note that the constant becomes $O(1) \delta$ in this case.

This completes the proof of the lemma.

From the lemma, we have $\delta_{k+1} \leqq M\left(\delta+\delta_{k}^{2}+\varepsilon \delta_{k}+\varepsilon \delta_{k}^{2}\right)$, for all $k$. Thus, if $4 \delta M^{2}+2 M \varepsilon+4 \delta M^{2} \varepsilon \leqq 1$, it follows by induction that $\delta_{k} \leqq 2 M \delta$ for all $k$. From this uniform bound, we obtain both convergence of the iteration scheme, and, in the limit, the desired bounds on the solution. 
Remark. With more care, the requirement that $\varepsilon$ be small can be removed in Proposition 3.4.1. Replacing $e^{-|x| / 2 C}$ by $(1 / \bar{\delta}) e^{-|x| / 2 C}$ in (3.34), (3.39), we have

$$
\begin{aligned}
\tilde{S}_{1}= & O(1)\left(\delta_{k}^{2}+\varepsilon \delta_{k}^{2}\right)\left[(t+1)^{-2} W_{C}(x, t+1)+K_{C}^{+}(x, t+1)^{2}+K_{C}^{+}(x, t+1)^{2}\right] \\
& +\left(\left[\delta_{k} / \bar{\delta}\right]^{2}+\varepsilon \delta_{k}\right)\left[(t+1)^{-1} e^{-|x| / 2 C}\right], \\
\tilde{S}_{1_{x}}= & O(1)\left(\delta_{k}^{2}+\varepsilon \delta_{k}^{2}\right)\left[t^{-1 / 2}(t+1)^{-3 / 2} W_{C}(x, t+1)+K_{C}^{+}(x, t+1)^{2}+K_{C}^{+}(x, t+1)^{2}\right] \\
& +\left(\left[\delta_{k} / \bar{\delta}\right]^{2}+\varepsilon \delta_{k}\right)\left[(t+1)^{-1} e^{-|x| / 2 C}\right] .
\end{aligned}
$$

Thus, (3.59) becomes

$$
\begin{aligned}
\hat{\hat{u}}_{k+1}= & O(1)\left(\delta_{k}^{2}+\varepsilon \delta_{k}^{2}+\varepsilon \bar{\delta} \delta_{k}+\bar{\delta}\left[\delta_{k} / \bar{\delta}\right]^{2}\right) \\
& \times\left[(t+1)^{-1} W_{C}(x, t+1)+(t+1)^{-1 / 2}(1 / \bar{\delta}) e^{-|x| / 2 C}\right], \\
\hat{\hat{u}}_{k+1_{x}}= & O(1)\left(\delta_{k}^{2}+\varepsilon \delta_{k}^{2}+\varepsilon \bar{\delta} \delta_{k}+\bar{\delta}\left[\delta_{k} / \bar{\delta}\right]^{2}\right) \\
& \times\left[t^{-1 / 2}(t+1)^{-1 / 2} W_{C}(x, t+1)+(t+1)^{-1 / 2} e^{-|x| / 2 C}\right] .
\end{aligned}
$$

If $\delta$ and $(\delta / \bar{\delta})$ are taken sufficiently small, then $\left(\delta_{k} / \bar{\delta}\right)$ remains small and the argument can be carried out as before.

5. Proof of the Main Theorem. At this point, we have merged completely with the analysis in [L-Zu]. The $L^{1}$ bounded stability result of Proposition 3.4.1 can be improved to the $L^{1}$ behavior given in Theorems 1 and 2 by exactly the bootstrap argument described in Sect. 5 of that reference, which we will not repeat.

\section{Concluding Discussion}

1. Generality. Among $2 \times 2$ systems (1-2), the example treated in Sect. 3 is more typical than might appear. Quadratic flux models such as (3.1) approximate the behavior of general $2 \times 2$ systems in the vicinity of a point of nonstrict hyperbolicity (cf. [Sch-Sh]). And, as discussed in [I-Ma-P1-T], a large class of undercompressive shock wave solutions of quadratic flux models are precisely straight line profiles of the form

$$
\Phi(x)=\left((1 / 2)\left(U_{-}+U_{+}\right)+(1 / 2) \tanh (\mu x)\left(U_{+}-U_{-}\right)\right),
$$

that is, scalar Burgers profiles along invariant lines of (3.1). Thus, it turns out that Eqs. (3.1-2) are representative of undercompressive shocks in a variety of systems.

2. Curved shocks. For quadratic models with $D=I$ and possessing only hyperbolic degeneracies, in fact all undercompressive shocks belong to the class of straight line profiles described above. This follows (cf. [I-Ma-Pl-T]) from the classification by canonical gradient models, [Sch-Sh], and a theorem of Chicone on saddle-saddle orbits of quadratic gradient dynamical systems.

However, for quadratic models, like (3.1-2), which possess parabolic degeneracies, there also exist curved-profile undercompressive shocks, in roughly equivalent number to the straight profile type [I-Ma-Pl]. Numerical study indicates that these 
shocks are also quite stable under perturbation [Zu-Pl-Ma.2]. It should be very interesting to study their nonlinear stability within the analytic framework set up in Sect. 2.

3. Extension to $n \geqq 3$. Additional complications can arise in $n \times n$ nonstrictly hyperbolic systems, $n \geqq 3$. However, the ideas of Sect. 2 are still applicable, with some elaboration.

Let $\Phi(x)=\Phi^{0}(x)$ be a viscous shock wave solution of (1) connecting endstates $\Phi_{ \pm \infty}$ and let $\Phi^{\alpha}$ parametrize the family of all such connections, $\alpha \in R^{j}$. The $j$-parameter family $\Phi^{\alpha}$ is the manifold of possible asymptotic states for a localized perturbation of $\Phi(x)$, generalizing the one-parameter family of translates of $\Phi$ that we considered in Sect. 2.

Likewise, in (2.6-7), the linearization of (1) around $\Phi(x)$, the functions $\frac{\partial}{\partial \alpha} \Phi_{\mid \alpha=0}^{\alpha}$ are stationary solutions representing instantaneous translation and/or deformation of $\Phi$, and generate a $j$-dimensional linear subspace of possible asymptotic states. Generically, this subspace contains all stationary solutions of the linearized equations.

To see this, we need only observe that the dynamical system

$$
D\left(\Phi_{ \pm \infty}\right)^{-1} f^{\prime}\left(\Phi_{ \pm \infty}\right) \bar{U}=\bar{U}_{x}
$$

describing stationary solutions of (2.6-7) can just as well be obtained by linearizing the dynamical system for stationary solutions of (1) around the particular solution $\Phi$. From this observation, it follows easily that the stable (resp. unstable) manifolds at $x= \pm \infty$ of the linearized equation (2.14) are given by the tangent space along $\Phi$ of the stable (resp. unstable) manifolds at $x= \pm \infty$ of the nonlinear traveling wave equations. Since the set of stationary solutions is the intersection of the unstable manifold at $x=-\infty$ with the stable manifold at $x=+\infty$, it has the same dimension $j$ for both the linearized and nonlinear equations, except in the degenerate case that the stable and unstable manifolds of the nonlinear equation are not transverse.

We will call the dimension $j$, counting the bounded solutions of (2.14), the degree of compressivity, $\# c$, of $\Phi$. Complementary to the manifold of bounded solutions are the invariant manifolds of solutions unbounded at both $\pm \infty$ and of solutions bounded at one end only, obtained by different intersections of the stable and unstable manifolds at $x= \pm \infty$. The dimension of the manifold of solutions unbounded at both ends we will call the degree of undercompressivity, $\# u$, and the dimension of the manifold of solutions with one-sided bound we will call the degree of transversality, \#t, of $\Phi$. It is clear from the definitions that

$$
\# c+\# u+\# t=n
$$

Save for this restriction, and the restriction that $\# c \geqq 1$ (necessary for existence of a shock profile), all possibilities can occur. This classification scheme refines the simpler counting of incoming and outgoing characteristics that suffices for the strictly hyperbolic case and for $n=2$. Proposition 2.2.1 becomes:

Proposition 4.3.1. Let $E$ denote the space of $L^{1}$ time-invariants of the linearized equations (2.6-7). Then, $\operatorname{dim}(E)=\# u+n$. 
Proof. We refer to the notation in Proposition 2.2.1, associating time-invariants with bounded solutions $e(x)$ of (2.11). Setting $W=e_{x}$, we have

$$
W f^{\prime}\left(\Phi_{ \pm \infty}\right) D^{-1}\left(\Phi_{ \pm \infty}\right)=W_{x}
$$

and $W \in L^{1}$.

But, solutions $W \in L^{1}$ of (2.16) can be characterized as those solutions which are orthogonal to the compressive and transverse manifolds of (2.14) at the point $x=0$. For, claim 2.1.2 implies that any such solution vanishes at $x= \pm \infty$, by the same argument used in Proposition 2.2.1.

The set of all such solutions $W$ has dimension $\# u$, by (4.2). The integration of $e_{x}=W$ introduces a further constant of integration, of dimension $n$, proving the result.

This proposition is quite significant. Presumably, the $L^{1}$ asymptotic state of a solution of the linearized equations (2.6-7) should consist of a stationary wave plus $(\# t+2 \# u)$ outgoing diffusion waves. Thus, we expect $(\# c+2 \# u+\# t)=(n+\# u)$ $L^{1}$ time-invariants if the shock is to be stable.

Further, we note that the invariants prescribing the deformations $\frac{\partial}{\partial \alpha_{l}} \Phi$ and the masses of the various diffusion waves are, generically, determined by $(\# t+2 \# u)$ boundary conditions analogous to (2.22) together with $(\# c)$ normalization conditions analogous to (2.23). More precisely, the invariants $e_{\alpha_{t}}$ corresponding to deformation $\frac{\partial}{\partial \alpha_{i}} \Phi$ satisfy

$$
e_{\alpha_{l}}( \pm \infty) r_{k}\left(\Phi_{ \pm \infty}\right)=0
$$

for all outgoing eigendirections $r_{k}$ and

$$
\left\langle e_{\alpha_{l}}(x), \frac{\partial}{\partial \alpha_{j}} \Phi\right\rangle=\delta_{j}^{l}
$$

for all $j$. The invariants $e_{k}$ corresponding to the mass of the outgoing diffusion wave in the $r_{k}$ direction satisfy

$$
e_{k}( \pm \infty) r_{l}\left(\Phi_{ \pm \infty}\right)=\delta_{l}^{k}
$$

for all outgoing eigendirections $r_{l}$ and

$$
\left\langle e_{k}(x), \frac{\partial}{\partial \alpha_{j}} \Phi\right\rangle=0
$$

for all $j$.

Thus, it is possible to define a diagonalizing transformation

$$
\tilde{U}=E(x) U
$$

similar to (2.27). We conjecture that (with appropriate conditions on the viscosity matrix $D(U)$ ) all weak shocks are stable in the orbital sense described above, so long as the matrix $E(x)$ is everywhere nonsingular.

Remark. A formal analogy between the relation we have observed between linearized and nonlinear stationary solutions, and the standard Melnikov integral has been pointed out by Dan Marchesin. 
4. Examples, $n=3$. To conclude, we give examples of the different types of undercompressive waves which are possible in the case $n=3$.

In each of these examples, the waves can be shown to be (orbitally) nonlinearly stable using the diagonalizing transformation and the analysis already performed in the proof of Theorem 1. Precisely, it can be shown that the $L^{1}$ asymptotic state of a perturbed shock $\Phi^{0}$ is a shock $\Phi^{\alpha}$ plus outgoing diffusion waves in each of the outgoing characteristic modes at $x= \pm \infty$.

Further, the instantaneous deformation $\alpha(t)$, defined analogously to $d(t)$ in 2.5 , evolves nonlinearly in the sense of Remark 2.3.1.

Example 1. Standard undercompressive shock $(\# c=1, \# u=1, \# t=1)$ :

$$
\begin{gathered}
\begin{cases}u_{t}+\frac{1}{2}\left(w^{2}-u^{2}\right)_{x}+\varepsilon v_{x} & =u_{x x} \\
v_{t}+(2 u v)_{x} & =v_{x x} \\
w_{t}+(2 w)_{x} & =w_{x x}\end{cases} \\
\Phi^{x}(x)=\left(\begin{array}{c}
\tanh \left(x-\alpha_{1}\right) \\
0 \\
0
\end{array}\right)
\end{gathered}
$$

This shock is undercompressive in one secondary field and transverse in the other. Example 2. Totally undercompressive shock $(\# c=1$, \#u=2, \#t=0):

$$
\begin{gathered}
\begin{cases}u_{t}+\frac{1}{2}\left(w^{2}-u^{2}\right)_{x}+\varepsilon v_{x} & =u_{x x} \\
v_{t}+(2 u v)_{x} & =v_{x x} \\
w_{t}+(u w)_{x} & =w_{x x}\end{cases} \\
\Phi^{\alpha}(x)=\left(\begin{array}{c}
\tanh \left(x-\alpha_{1}\right) \\
0 \\
0
\end{array}\right)
\end{gathered}
$$

This shock is undercompressive in both secondary fields.

Example 3. Mixed over/undercompressive shock $(\# c=2, \# u=1, \# t=0)$ :

$$
\begin{gathered}
\left\{\begin{array}{cl}
u_{t}+\frac{1}{2}\left(w^{2}-u^{2}\right)_{x}+\varepsilon v_{x} & =u_{x x} \\
v_{t}+\left(2 w^{2}-v^{2}\right)_{x} & =v_{x x} \\
w_{t}+(u w)_{x} & =w_{x x}
\end{array}\right. \\
\Phi^{\alpha}(x)=\left(\begin{array}{c}
\tanh \left(x-\alpha_{1}\right) \\
\tanh \left(x-\alpha_{2}\right) \\
0
\end{array}\right)
\end{gathered}
$$

This shock is doubly compressive, meaning that there exists a two-parameter family of traveling waves connecting the same endstates. It has a single, undercompressive secondary field. Its behavior has qualities of both overcompressive and undercompressive shocks. Namely, the shock is only stable within the family of possible deformations, as typical of overcompressive shocks, [F-L, L.4], while the asymptotic state evolves nonlinearly, as typical of undercompressive shocks (Sect. 2). 
Finally, we remark that, according to the classical, hyperbolic characteristic condition, this would appear to be a standard Lax shock. Once again, this illustrates the importance of parabolic effects in the behavior of nonstrictly hyperbolic waves.

Acknowledgements. Kevin Zumbrun thanks the Stanford University Mathematics Department for its hospitality during the visit in which this paper was begun. Thanks also to Vicki Botos at Indiana University, for her efforts in typing the manuscript, and to Dan Marchesin and Brad Plohr for stimulating discussions. This work was supported in part by NSF grant DMS-9107990 (Zumbrun), and by NSF grant DMS 90-0226 and Army grant DAAL03-87-K-0063 (Liu).

\section{References}

[A-Ga-J] Alexander, J., Gardner, R., Jones, C.K.R.T.: A topological invariant arising in the analysis of traveling waves. J. Reine Angew. Math. 410, 167-212 (1990)

[Che-L] Chern, I.L., Liu, T.P.: Convergence to diffusion waves of solutions for viscous conservation laws. Commun. Math. Phys. 110, 503-517 (1987), Erratum 120, 525-527 (1989)

[Che-X] Chern, I.L., Xin, Z.: Stability of inviscid complex Burgers shock. Unpublished calculation (1989)

[Co-H] Courant, R., Hilbert, D.: Methods of mathematical physics, VII. New York. Interscience, 1953

[E] Evans, J.W.: Ind. Univ. Math. J. 21, 877-955, 75-90, and 577-594; (1972) 24, $1169-1190(1975)$

[F.1] Freistuhler, H.: Dynamical stability and vanishing viscosity: A case study of a nonstrictly hyperbolic system of conservation laws. CPAM 45, 561-582 (1992)

[F-L] Freistuhler, H., Liu, T.P.: Nonlinear stability of overcompressive shock waves in a rotationally invariant system of viscous conservation laws. Commun. Math. Phys. 153, 147-158 (1993)

[G.1] Goodman, J.: Nonlinear asymptotic stability of viscous shock profiles for conservation laws. Arch. Rat. Mech. 95, 325-344 (1986)

[G.2] Goodman, J.: Remarks on the stability of viscous shock waves, Viscous profiles and numerical methods for shock waves. Ed: Shearer, M., Philadelphia, PA: SIAM, 1991, pp. 66-72

[G.3] Goodman, J.: Viscous scalar shock fronts in several dimensions. Preprint (1991)

[H-Sm.1] Hoff, D., Smoller, J.: Solutions in the large for certain nonlinear parabolic systems. Ann. Inst., Henri Poincaré 2.3, 210-220 (1985)

[I-Ma-P1] Isaacson, E., Marchesin, D., Plohr, B.: Transitional waves for conservation laws. SIAM J. Math. Anal. V 21, 837-866 (1990)

[Ka] Kapitula, T.: On the stability of travelling waves in weighted $L^{\infty}$ spaces. Preprint (1992).

[L.1] Liu, T.P.: Nonlinear stability of shock waves for viscous conservation laws. Mem. Amer. Math. Soc. V 56, 328, 1-108 (1985)

[L.2] Liu, T.P.: Decay to N-waves of solutions of general systems of nonlinear hyperbolic conservation laws. Comm. Pure Appl. Math. V 30, 585-610 (1977)

[L.3] Liu, T.P.: Pointwise convergence to viscous shock wave solutions of conservation laws. To appear

[L.4] Liu, T.-P.: Interactions of Hyperbolic waves, Viscous profiles and numerical methods for shock waves. Ed: Shearer, M., Philadelphia, PA: SIAM, pp. 66-72, 1991

[L-Ze] Liu, T.P., Zeng, Y.: Pointwise convergence to diffusion waves for solutions of a viscous conservation law. To appear

[Maj-Pe] Majda, A., Pego, R.: Stable viscosity matrices for system of conservation laws. J. Differential Equations V 56, 229-262 (1985)

[Mat-Ni] Matsumura, A., Nishihara, K.: On the stability of travelling wave solutions of a one dimensional model system for compressible viscous gas. Japan J. Appl. Math. 2, $17-25(1985)$ 
[Ma-Me] Marchesin, D., Medeiros, H.: A note on the stability of eigenvalue degeneracy in nonlinear conservation laws of multiphase flow. Current Progress in Hyperbolic Systems: Riemann Problems and Computations (Bowdoin, 1988) Contemporary Mathematics V 100, Lindquist B., Providence, RI: American Mathematics Society, pp. 215-223 1989

[Sat] Sattinger, D.: On the stability of waves of nonlinear parabolic systems. Adv. Math. V 22, 312-355 (1976)

[Sch-Sh] Schaeffer, D., Shearer, M.: The classification of $2 \times 2$ systems of non-strictly hyperbolic conservation laws, with application to oil recovery. J Comm. Pure Appl. Math. V 40, 141-178 (1987)

[Sz-X] Szepessy, A., Xin, Z.P.: Nonlinear stability of viscous shock waves. Report TRITANA-9201, Royal Institute of Technology, S-100 44 Stockholm (1992)

[Ze] Zeng, Y.: Convergence to diffusion waves of solutions to nonlinear viscoelastic model with fading memory. Commun. Math. Phys. 146, 585-609 (1992)

[Zu.1] Zumbrun, K.: Formation of diffusion waves in a scalar conservation law with convection. To appear, AMS Proc. (1993)

[Zu.2] Zumbrun, K.: Stability of general inviscid shock. Unpublished calculation (1990)

[Zu-Pl-Ma] Zumbrun, K., Plohr, B., Marchesin, D.: Scattering behavior of transitional shock waves. Mat. Contemp. V 3, 191-209 (1992)

[Zu-Pl-Ma.2] Zumbrun, K., Plohr, B., Marchesin, D.: Unpublished computations

Communicated by A. Jaffe. 
